\title{
LMP2 expression and proteasome activity in NOD mice
}

To the Editor- The non-obese diabetic (NOD) mouse is an established and widely used model of autoimmunetype I diabetes $^{1}$. Besides the involvement of class II-and class I-restricted T cells, additional major histocompatibility complex ( $\mathrm{MHC}$ ) and non-M HC-linked genetic factors contribute to disease susceptibility ${ }^{2}$. Faustman and colleagues claim that in NOD mice the expression of the IFN $\gamma$-inducible large multifunctional proteasome subunit 2 (LMP2; Genome Database designation, PSMB9) is de creased both at the mRNA and protein level ${ }^{3,4}$. In addition, they noted a failure to appropriately activate $\mathrm{NF \kappa B}$ in cells from NOD mice due to defective $I \kappa B$ degradation. This observation was attributed to the claimed defect in LMP2 synthesis. While the published data show that activation of NFKB relies on proteasomal proteolysis, there is no independent evidence that such activation would require the specific involvement of LMP2-bearing proteasomes ${ }^{5}$.

We examined the expression of LMP2 mRNA by real time PCR and found no evidence for impaired transcription of the LMP2 gene in NOD mice as compared to BALB/C mice (Fig. 1a). Further, immunoblot analysis showed no reduction of LMP2, LMP7 and MECL1 protein levels in NOD mice, compared to other strains (Fig. 1b). The slight differences in mobility of LMP2 and LMP7 proteins on SDS PAGE are attributable to their ge netic polymorphism. What about proteasome activity? The assessment of proteasomal proteolysis in crude extracts

Fig. 1 Normal LMP2 expression and proteasome function in NOD mice. a, Quantitation of LMP2 mRNA levels in spleen from 12 week old NOD and BALB/C mice by real-time RTPCR using the ABI Prism 7700 (Appl. Biosystems). LMP2 levels were determined from five replicates and normalized to the average of GAPDH mRNA levels in each sample. For NOD and BALB/C mice, total mRNA from two mice and three mice, respectively, was pooled and used for analysis. b, Immunoblot analysis of LMP2, LMP7 and MECL1 immunoproteasome $\beta$ subunit proteins. Cell extracts were prepared from $\mathrm{BALB} / \mathrm{C}, \mathrm{B} 6, \mathrm{C} 3 \mathrm{H}$ and NOD spleens and analysed as described ${ }^{6}$ c,

Proteasome activity assessed by the radiolabeled active site-directed probe $\left.{ }^{125} \mathrm{I}\right]-\mathrm{NLVS}$ in extracts from spleen, thymus and brain was performed as

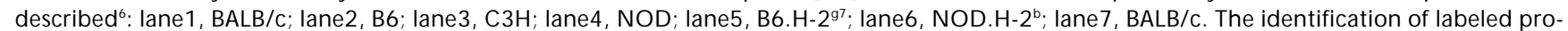
teasome $\beta$ subunits was based on previous studies ${ }^{6}$. Note the absence of labeled LMP2 in brain cell extracts, which do not express immunoproteasomes (lane7). using fluorogenic substrates is complicated by the presence of other non-proteasomal peptidases. We therefore analyzed directly the content of cell extracts for active proteasome $\beta$ subunits using a mechanism-based active site directed probe 6 . We observed normal proteasome activity in extracts from thymus and spleen of NOD mice (Fig. 1c). Active immunoproteasomes including LMP2 are present at comparable levels in all strains analyzed, and did not appear to be influenced by age, gender or the presence or absence of diabetes (Fig. 1c and data not shown). LMP2, together with LMP7 and MECL1 are essential subunits for the assembly of the immunoproteasome ${ }^{7}$. Mere overexpression of a single immunoproteasome subunit does not lead to assembly of functional immunoproteasomes, and conversely, the ablation of either LMP2 or LMP7 suffices to eliminate formation of immunoproteasomes which contain all three interferon- $\gamma$-inducible $\beta$ subunits ${ }^{8}$. Thus, the absence of LMP2, as claimed by Faustman and coworkers, should have abolished the activity of immunoproteasomes al together.

In summary, our data show normal LMP2 expression and proteasome activity in NOD mice. Consequently, there is no reason to entertain the possibility that compromised proteasomal function would cause diabetes in NOD mice. A failure to activate NFKB is not addressed by our analysis and, if confirmed independently, remains a factor that could contribute to the NOD phenotype.

\section{Acknowledgments}

This work was supported in part by the Juvenile Diabetes Foundation International through the Juvenile Diabetes Foundation Center for Islet Transplantation at HarvardM edical School. We thank D.M athis and C.Benoist from the Joslin Diabetes center for sharing the $\mathrm{B} 6 . \mathrm{H}-2^{97}$ mice. B.M.K was supported by a long-term fellowship from the Human Frontier Science Program Organisation, and A.-M.L.-D. by a Juvenile Diabetes Foundation postdoctoral fellowship.

Benedikt M. KeSSLeR ${ }^{1}$, Ana-M aria LENNON-DUMÉNIL ${ }^{1}$, MARI L. SHINOHARA ${ }^{2}$, MYRA A. LIPES ${ }^{2}$ AND HIDDE L. PLOEGH ${ }^{1}$

${ }^{1}$ Department of Pathology, Harvard Medical School, 200 Longwood Avenue, Boston, M assachusetts 02115, USA ${ }^{2}$ Research Division, Joslin Diabetes Center, Harvard M edical School, Boston, Massachusetts 02215, USA B.M.K \& A.L.D contributed equally to this work Correspondence should be addressed to H.L.P.

To the Editor-Hayashi and Faustman ${ }^{4}$ re port that diabetes-prone NOD mice are defective in expression of LMP2, and that this defect in proteasome subunit composition leads to an inability to proteolytically process the NF-kB p105 precursor into its mature form, p50. They conclude that the proteasome subunits encoded by the MHC are obligatory in NF-kB processing. Contrary to the report by Hayashi and Faustman, we find that NOD mice have normal expression levels of LMP2, and that p50 production does a

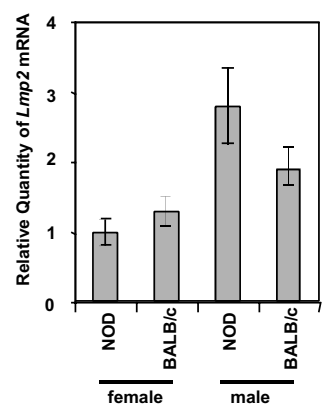

b

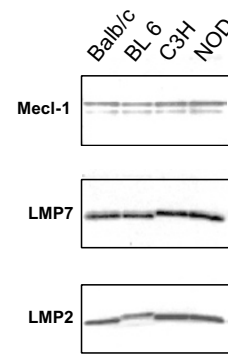

Spleen
C [125]-NLVS

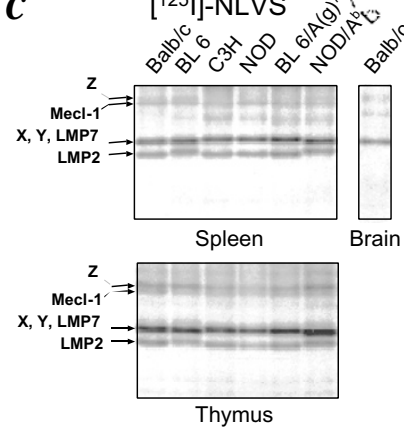

